Document downloaded from:

http://hdl.handle.net/10251/64697

This paper must be cited as:

Argyros, IK.; Cordero Barbero, A.; Magreñán Ruiz, AA.; Torregrosa Sánchez, JR. (2015). On the convergence of a damped-secant method with modified right-hand side vector. Applied Mathematics and Computation. 252:315-323. doi:10.1016/j.amc.2014.12.029.

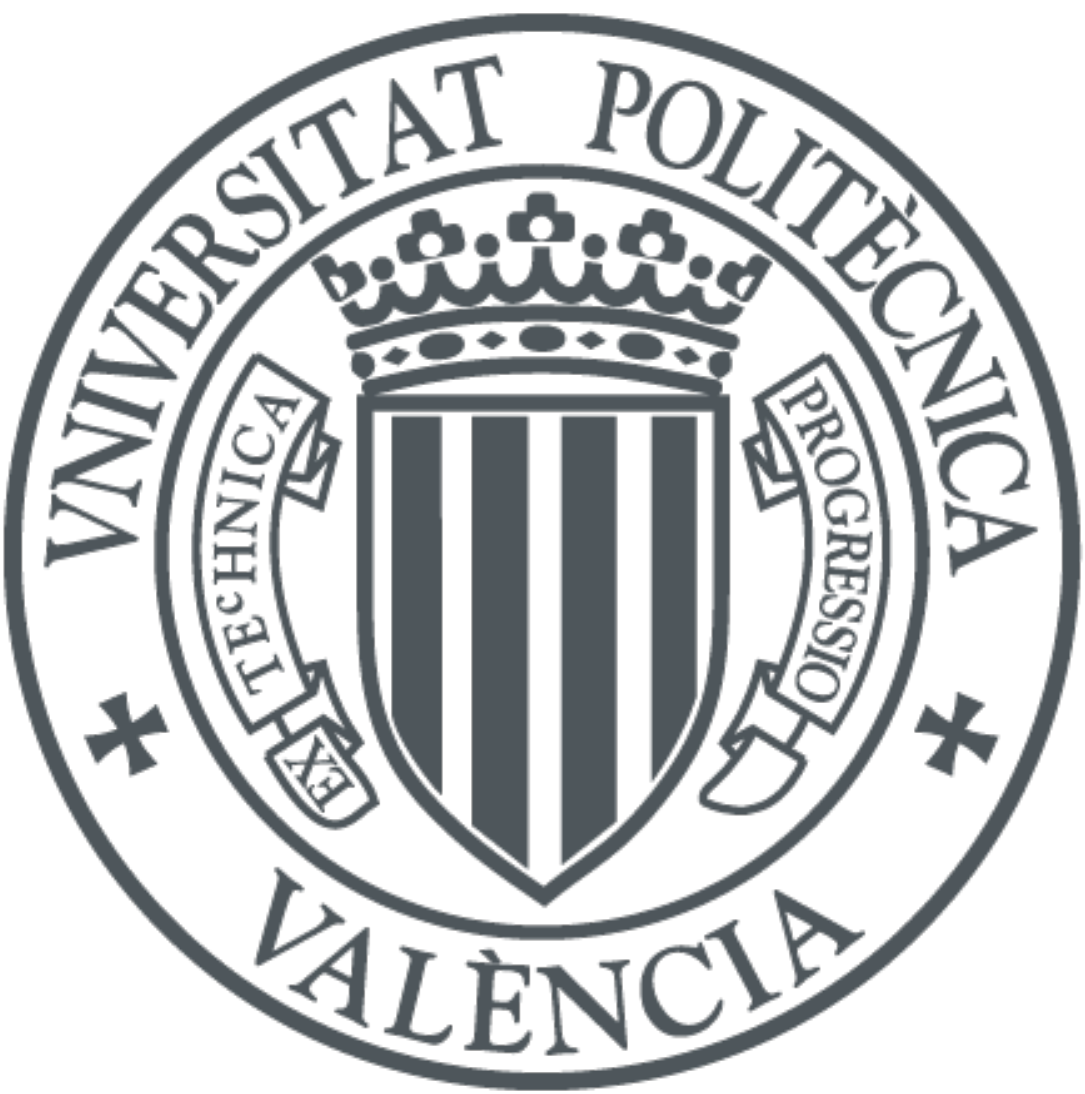

The final publication is available at

http://dx.doi.org/10.1016/j.amc.2014.12.029

Copyright Elsevier

Additional Information 


\title{
On the convergence of a Damped Secant method with modified right-hand side vector*
}

\author{
Ioannis K. Argyros ${ }^{a}$, Alicia Cordero $^{b}$, Á. Alberto Magreñán ${ }^{c}, J_{. R}$. Torregrosa ${ }^{b}$ \\ ${ }^{a}$ Department of Mathematics Sciences, Cameron University, \\ Lawton, OK 73505, USA \\ iargyros@cameron.edu \\ ${ }^{b}$ Instituto de Matemática Multidisciplinar, Universitat Politècnica de València, \\ Camino de Vera, s/n, 46022 València, Spain \\ \{acordero, jrtorre\}@mat.upv.es \\ ${ }^{c}$ Departmento de Matemáticas y Computación, Universidad de La Rioja, \\ 26002 Logrño, Spain \\ alberto.magrenan@gmail.com
}

\begin{abstract}
We present a convergence analysis for a Damped secant method with modified right-hand side vector in order to approximate a locally unique solution of a nonlinear equation in a Banach spaces setting. In the special case when the method is defined on $\mathbb{R}^{i}$, our method provides computable error estimates based on the initial data. Such estimates were not given in relevant studies such as $[12,13]$. Numerical examples further validating the theoretical results are also presented in this study.
\end{abstract}

Keywords:Damped Secant method, Banach space, local/semi-local convergence

AMS 2010:65H10, 65G99, 49M15

\section{Introduction}

In this study we are concerned with the problem of approximating a locally unique solution $x^{*}$ of the nonlinear equation

$$
F(x)=0,
$$

where $F$ is a Fréchet-differentiable operator defined on a open convex subset $D$ of a Banach space $\mathbb{X}$ with values in a Banach space $\mathbb{Y}$.

Many problems from Computational Sciences and other disciplines can be brought in a form similar to equation (1.1) using Mathematical Modeling [3, 8, 7, 14, 11]. For example in data fitting, we have $\mathbb{X}=\mathbb{Y}=\mathbb{R}^{i}$, $i$ is number of parameters and observations.

The solution of (1.1) can rarely be found in closed form. That is why most solution methods for these equations are usually iterative. In particular, the practice of Numerical Analysis for finding such solutions is essentially connected to Newton-like methods $[3,5,6,7,14,15,16]$. The study about convergence matter of iterative procedures is usually centered on two types: semilocal and local convergence analysis. The semilocal convergence matter is, based on the information around an initial point, to give criteria ensuring the convergence of iteration procedures; while the local one is, based on the information around a solution, to find estimates of the radii of the convergence balls. Local and semilocal convergence of Newton-like methods as well as an error analysis for such methods can be found in $[1,2,5,7,9,10]$.

*This research was supported by Ministerio de Ciencia y Tecnología MTM2011-28636-C02-\{01,02\} and Universitat Politècnica de València SP20120474. 
In the present paper, we study the convergence of the Damped Secant method defined by

$$
x_{n+1}=x_{n}-A^{-1}\left(I-\alpha_{n}\left(\left[x_{n-1}, x_{n} ; F\right]-A\right)\right) F\left(x_{n}\right), \text { for each } n=0,1,2, \ldots,
$$

where $x_{-1}$ and $x_{0}$ are initial points, $A \in \mathcal{L}(\mathbb{X}, \mathbb{Y})$ the space of bounded linear operators from $\mathbb{X}$ into $\mathbb{Y}$, $A^{-1} \in \mathcal{L}(\mathbb{Y}, \mathbb{X}),[x, y ; F] \in \mathcal{L}(\mathbb{X}, \mathbb{Y})$ is a divided difference of order one at the point $(x, y)$ with $x, y \in D$ and $\alpha_{n}$ is a sequence of real numbers chosen to force convergence of sequence $x_{n}$.

If the divided difference $[x, y ; F]$ is replaced by $F^{\prime}(x)$ we get

$$
z_{n+1}=z_{n}-A^{-1}\left(I-\alpha_{n}\left(F^{\prime}\left(z_{n}\right)-A\right)\right) F\left(z_{n}\right) \text { for each } n=0,1,2, \ldots,
$$

The local convergence of the method (1.3) was studied by Krejić and Lužanin [13] (see also [12]) in the case when $\mathbb{X}=\mathbb{Y}=\mathbb{R}^{i}$.

If $A=\left[x_{n-1}, x_{n} ; F\right]$ and $\alpha_{n}=0$ for each $n=0,1,2, \ldots$, we obtain the secant method

$$
x_{n+1}=x_{n}-\left[x_{n-1}, x_{n} ; F\right]^{-1} F\left(x_{n}\right), \text { for each } n=0,1,2, \ldots,
$$

If $A=\left[x_{-1}, x_{0} ; F\right]$ and $\alpha_{n}=0$ for each $n=0,1,2, \ldots$, we obtain the modified-secant method

$$
x_{n+1}=x_{n}-\left[x_{-1}, x_{0} ; F\right]^{-1} F\left(x_{n}\right), \text { for each } n=0,1,2, \ldots,
$$

It is well-known that Newton's method

$$
x_{n+1}=x_{n}-F^{\prime}\left(x_{n}\right) F\left(x_{n}\right), \text { for each } n=0,1,2, \ldots,
$$

converges quadratically provided that the iteration starts close enough to the solution. However, the cost of a Newton iterate may be very expensive, since all the elements of the Jacobian matrix involved must be computed, as well as the need for an exact slowdown of a system of linear equations using a new matrix for every iterate. As noted in [13] Newton-like method (1.3) uses a modification of the right hand side vector, which is cheaper than the Newton and faster than the modified Newton method. One step of the method requires the solution of a linear system, but the system matrix is the same in all iterations.

Similar problems we have if we use the secant method (1.4) which converges with order $1.618 \ldots<2$. That is why Damped secant method is a good alternative to all the preceding methods, especially to the method (1.3) considered in [13].

We present a local and semilocal convergence analysis for Damped Secant method (1.2). In the local case the radius of convergence can be computed as well as the error bounds on the distances $\left\|x_{n}-x^{*}\right\|$ for each $n=0,1,2, \ldots$. In the semilocal case, we provide estimates on the smallness of $\left\|F\left(x_{0}\right)\right\|$ as well as computable estimates for $\left\|x_{n}-x^{*}\right\|$ (not given in $[12,13]$ in terms of the Lipschitz constants and other initial data).

We denote by $U(\nu, \mu)$ the open ball centered at $\nu \in \mathbb{X}$ and of radius $\mu>0$. Moreover, by $\overline{U(\nu, \mu)}$ we denote the closure of $U(\nu, \mu)$.

The paper is organized as follows. Sections 2 and 3 contain the semilocal and local convergence analysis of Secant-like method (1.2), respectively. The numerical examples are presented in the concluding Section 4.

\section{Semilocal convergence}

In this section we present the semilocal convergence of Damped Secant method (1.2). We shall use the following conditions:

$\left(C_{0}\right) F: D \subseteq \mathbb{X} \rightarrow \mathbb{Y}$ is Fréchet-differentiable and there exists $A \in \mathcal{L}(\mathbb{X}, \mathbb{Y})$ such that $A^{-1} \in \mathcal{L}(\mathbb{Y}, \mathbb{X})$ with $\left\|A^{-1}\right\| \leq a$

$\left(C_{1}\right)$ There exists $L>0$ such that for each $x, y \in D$ the Lipschitz condition

$$
\left\|F^{\prime}(x)-F^{\prime}(y)\right\| \leq L\|x-y\|
$$

holds; 
$\left(C_{2}\right)$ Given $x_{0} \in D$, there exist $L_{0}>0, a_{0} \geq 0$ and $a_{1} \geq 0$ such that for each $x \in D$ the center-Lipschitz condition

$$
\left\|F^{\prime}(x)-F^{\prime}\left(x_{0}\right)\right\| \leq L_{0}\left\|x-x_{0}\right\|,
$$

$\left\|F^{\prime}\left(x_{0}\right)-A\right\| \leq a_{0}$ and $\left\|A^{-1}\left(F^{\prime}\left(x_{0}\right)-A\right)\right\| \leq a_{1}$ hold;

$\left(C_{3}\right)$ There exist a divided difference $[x, y ; F]$ and $L_{1} \geq 0$ such that for each $x, y \in D$

$$
\left\|[x, y ; F]-F^{\prime}\left(x_{0}\right)\right\| \leq L_{1}\left(\left\|x-x_{0}\right\|+\left\|y-x_{0}\right\|\right) ;
$$

$\left(C_{4}\right)$ There exists $\alpha \geq 0$ and $c>0$ such that

$$
\left|\alpha_{n}\right| \leq \alpha \quad \text { and } \quad\left\|x_{-1}-x_{0}\right\| \leq c, x_{-1} \in D .
$$

$\left(C_{5}\right)$ There exists $q \in(0,1)$ such that

$$
a+\alpha\left(a_{1}+\frac{2 a L_{1} q\left\|F\left(x_{0}\right)\right\|}{1-q}\right) \leq q
$$

and

$$
\frac{L}{2} q^{2}\left\|f\left(x_{0}\right)\right\|+\left(\frac{L_{0} q\left\|F\left(x_{0}\right)\right\|}{1-q}+a_{0}\right) q+\alpha\left(a_{0}+\frac{2 L_{1} q\left\|F\left(x_{0}\right)\right\|}{1-q}\right) \leq q ;
$$

$\left(C_{6}\right)$ There exists $q \in(0,1)$ such that the first inequality in $\left(C_{5}\right)$ holds and also

$$
\left(\frac{2}{1-q}+\frac{1}{2}\right) L_{0} q\left\|F\left(x_{0}\right)\right\|+\left(\frac{L_{0} q\left\|F\left(x_{0}\right)\right\|}{1-q}+a_{0}\right) q+\alpha\left(a_{0}+\frac{2 L_{1} q\left\|F\left(x_{0}\right)\right\|}{1-q}\right) \leq q
$$

$\left(C_{7}\right) \overline{U\left(x_{0}, r\right)} \subseteq D$, with $r=\frac{q\left\|F\left(x_{0}\right)\right\|}{1-q}$.

Notice that $\left(C_{1}\right)$ implies $\left(C_{2}\right)$,

$$
L_{0} \leq L
$$

holds in general and $\frac{L}{L_{0}}$ can be arbitrarily large $[3,4,7]$. The conditions involving $\left\|F\left(x_{0}\right)\right\|$ and $q$ in $\left(C_{5}\right)$ and $\left(C_{6}\right)$ can be solved for $\left\|F\left(x_{0}\right)\right\|$ and $q$. However, these representations are very long and unattractive. That is why we decided to leave these conditions as uncluttered as possible. Notice also that these conditions determine the smallness of $\left\|F\left(x_{0}\right)\right\|$ and $q$. From now on we shall denote $\left(C_{0}\right)-\left(C_{5}\right),\left(C_{7}\right)$ and $\left(C_{0}\right),\left(C_{2}\right)-\left(C_{4}\right),\left(C_{6}\right)-\left(C_{7}\right)$ as the $(C)$ and $\left(C^{0}\right)$ conditions, respectively. Next, we present the semilocal convergence of the Damped Secant-like method (1.2) first under the $(C)$ conditions.

Theorem 2.1 Suppose that the $(C)$ conditions hold. Then sequence $\left\{x_{n}\right\}$ generated by the Damped Secant method (1.2) is well defined, remains in $\overline{U\left(x_{0}, r\right)}$ for each $n=0,1,2, \ldots$, and converges to a solution $x^{*} \in$ $\overline{U\left(x_{0}, r\right)}$ of equation (1.1). Moveover, the following estimates hold for each $n=0,1,2, \ldots$,

$$
\left\|x_{n+1}-x_{n}\right\| \leq q\left\|F\left(x_{n}\right)\right\| \leq q^{n+1}\left\|F\left(x_{0}\right)\right\|,
$$

and

$$
\left\|F\left(x_{n+1}\right)\right\| \leq q\left\|F\left(x_{n}\right)\right\| \leq q^{n+1}\left\|F\left(x_{0}\right)\right\|,
$$

where $q$ is defined in $\left(C_{5}\right)$ and $r$ in $\left(C_{7}\right)$. 
Proof. By constructing (1.2) and $A^{-1} \in \mathcal{L}(\mathbb{Y}, \mathbb{X})$, sequence $\left\{x_{n}\right\}$ is well defined. Then, we shall show that $x_{1} \in U\left(x_{0}, r\right),\left\|x_{1}-x_{0}\right\| \leq q\left\|F\left(x_{0}\right)\right\|$ and $\left\|F\left(x_{1}\right)\right\| \leq q\left\|F\left(x_{0}\right)\right\|$. Indeed, we have by (1.2) for $n=0$ and conditions in $\left(C_{0}\right),\left(C_{2}\right),\left(C_{3}\right),\left(C_{4}\right),\left(C_{5}\right)$ and $\left(C_{7}\right)$ that

$$
\begin{aligned}
\left\|x_{1}-x_{0}\right\| & =\left\|A^{-1}\left(I-\alpha_{0}\left(\left[x_{-1}, x_{0} ; F\right]-A\right)\right) F\left(x_{0}\right)\right\| \\
& \leq\left[\left\|A^{-1}\right\|+\left|\alpha_{0}\right|\left[\left\|A^{-1}\right\|\left\|\left[x_{-1}, x_{0} ; F\right]-F^{\prime}\left(x_{0}\right)\right\|+\left\|A^{-1}\left(F^{\prime}\left(x_{0}\right)-A\right)\right\|\right]\right]\left\|F\left(x_{0}\right)\right\| \\
& \leq\left[a+\alpha\left(a_{1}+a L_{1} c\right)\right]\left\|F\left(x_{0}\right)\right\| \\
& \leq q\left\|F\left(x_{0}\right)\right\|<r .
\end{aligned}
$$

Hence, $x_{1} \in U\left(x_{0}, r\right)$ and (2.4) holds for $n=0$. Using (1.2) and some algebraic manipulation, we obtain the Ostrowski-type approximation

$$
\begin{aligned}
F\left(x_{n+1}\right)= & \int_{0}^{1}\left[F^{\prime}\left(x_{n}+\theta\left(x_{n+1}-x_{n}\right)\right)-F^{\prime}\left(x_{n}\right)\right]\left(x_{n+1}-x_{n}\right) \mathrm{d} \theta \\
& +\left(F^{\prime}\left(x_{n}\right)-F^{\prime}\left(x_{0}\right)+F^{\prime}\left(x_{0}\right)-A\right)\left(x_{n+1}-x_{n}\right)+ \\
& \alpha_{n}\left(\left[x_{n-1}, x_{n} ; F\right]-F^{\prime}\left(x_{0}\right)+F^{\prime}\left(x_{0}\right)-A\right) F\left(x_{n}\right) .
\end{aligned}
$$

Using (2.6), and the $(C)$ conditions, for $n=0$ we get in turn that

$$
\begin{aligned}
\left\|F\left(x_{1}\right)\right\| \leq & \int_{0}^{1}\left\|F^{\prime}\left(x_{0}+\theta\left(x_{1}-x_{0}\right)\right)-F^{\prime}\left(x_{0}\right)\right\|\left\|x_{1}-x_{0}\right\| \mathrm{d} \theta \\
& +\left\|F^{\prime}\left(x_{0}\right)-A\right\|\left\|x_{1}-x_{0}\right\|+\left|\alpha_{n}\right|\left(\left\|\left[x_{-1}, x_{0} ; F\right]-F^{\prime}\left(x_{0}\right)\right\|+\left\|F^{\prime}\left(x_{0}\right)-A\right\|\right)\left\|F\left(x_{0}\right)\right\| \\
\leq & \frac{L_{0}}{2}\left\|x_{1}-x_{0}\right\|^{2}+a_{0} q\left\|F\left(x_{0}\right)\right\|+\alpha\left(a_{0}+L_{1} c\right)\left\|F\left(x_{0}\right)\right\| \\
\leq & \frac{L}{2} q^{2}\left\|F\left(x_{0}\right)\right\|^{2}+a_{0} q\left\|F\left(x_{0}\right)\right\|+\alpha\left(a_{0}+L_{1} c\right)\left\|F\left(x_{0}\right)\right\| \\
\leq & {\left[\frac{L}{2} q^{2}\left\|F\left(x_{0}\right)\right\|+a_{0} q+\alpha\left(a_{0}+L_{1} c\right)\right]\left\|F\left(x_{0}\right)\right\| } \\
\leq & q\left\|F\left(x_{0}\right)\right\| .
\end{aligned}
$$

That is (2.5) holds for $n=0$. Using (1.2) for $n=1$, we get by $\left(C_{0}\right),\left(C_{2}\right),\left(C_{3}\right),\left(C_{4}\right)$ and $\left(C_{5}\right)$ that

$$
\begin{aligned}
\left\|x_{2}-x_{1}\right\| & =\left\|A^{-1}\left(I-\alpha_{1}\left(\left[x_{0}, x_{1} ; F\right]-A\right)\right) F\left(x_{1}\right)\right\| \\
& \leq\left[\left\|A^{-1}\right\|+\left|\alpha_{1}\right|\left(\left\|A^{-1}\right\|\left(F^{\prime}\left(x_{0}\right)-A\right)\|+\| A^{-1}\|\|\left[x_{0}, x_{1} ; F\right]-F^{\prime}\left(x_{0}\right) \|\right)\right]\left\|F\left(x_{1}\right)\right\| \\
& \leq\left[a+\alpha\left(a_{1}+a L_{1}\left\|x_{1}-x_{0}\right\|\right)\right]\left\|F\left(x_{1}\right)\right\| \\
& \leq\left[a+\alpha\left(a_{1}+a L_{1} q\left\|F\left(x_{0}\right)\right\|\right)\right]\left\|F\left(x_{1}\right)\right\| \\
& \leq q\left\|F\left(x_{1}\right)\right\| \leq q^{2}\left\|F\left(x_{0}\right)\right\| .
\end{aligned}
$$

We also have that, by $\left(C_{7}\right)$

$$
\begin{aligned}
\left\|x_{2}-x_{0}\right\| & \leq\left\|x_{2}-x_{1}\right\|+\left\|x_{1}-x_{0}\right\| \\
& \leq q^{2}\left\|F\left(x_{0}\right)\right\|+q\left\|F\left(x_{0}\right)\right\| \\
& =q\left\|F\left(x_{0}\right)\right\| \frac{1-q^{2}}{1-q} \\
& <\frac{q\left\|F\left(x_{0}\right)\right\|}{1-q}=r .
\end{aligned}
$$


That is, $x_{2} \in U\left(x_{0}, r\right)$. Then, using $(2.6)$ for $n=1$, as above we get in turn that by the $(C)$ conditions,

$$
\begin{aligned}
\left\|F\left(x_{2}\right)\right\| \leq & \frac{L}{2}\left\|x_{2}-x_{1}\right\|^{2}+\left(L_{0}\left\|x_{1}-x_{0}\right\|+a_{0}\right)\left\|x_{2}-x_{1}\right\|+ \\
& \alpha\left(a_{0}+L_{1}\left(\left\|x_{n-1}-x_{0}\right\|+\left\|x_{n}-x_{0}\right\|\right)\right)\left\|F\left(x_{1}\right)\right\|+\frac{L}{2} q^{3}\left\|F\left(x_{0}\right)\right\|\left\|F\left(x_{1}\right)\right\|+ \\
& \left(L_{0} q\left\|F\left(x_{0}\right)\right\|+a_{0}\right) q\left\|F\left(x_{1}\right)\right\|+\alpha\left(a_{0}+2 L_{1} \frac{q\left\|F\left(x_{0}\right)\right\|}{1-q}\right)\left\|F\left(x_{1}\right)\right\| \\
\leq & {\left[\frac{L}{2} q^{3}\left\|F\left(x_{0}\right)\right\|+\left(L_{0} q\left\|F\left(x_{0}\right)\right\|+a_{0}\right) q+\alpha\left(a_{0}+2 L_{1} \frac{q\left\|F\left(x_{0}\right)\right\|}{1-q}\right)\right]\left\|F\left(x_{1}\right)\right\| } \\
\leq & q\left\|F\left(x_{1}\right)\right\| \leq q^{2}\left\|F\left(x_{0}\right)\right\| .
\end{aligned}
$$

Similarly, we have

$$
\begin{aligned}
\left\|x_{3}-x_{2}\right\| & =\left\|A^{-1}\left(I-\alpha_{2}\left(\left[x_{1}, x_{2} ; F\right]-A\right)\right) F\left(x_{2}\right)\right\| \\
& \leq\left[\left\|A^{-1}\right\|+\left|\alpha_{2}\right|\left(\left\|A^{-1}\left(F^{\prime}\left(x_{0}\right)-A\right)\right\|+\left\|A^{-1}\right\|\left(\left[x_{1}, x_{2} ; F\right]-F^{\prime}\left(x_{0}\right)\right)\right)\right]\left\|F\left(x_{2}\right)\right\| \\
& \leq\left[a+\alpha\left(a_{1}+a L_{1}\left(\left\|x_{1}-x_{0}\right\|+\left\|x_{2}-x_{0}\right\|+\right)\right)\right]\left\|F\left(x_{2}\right)\right\| \\
& \leq\left[a+\alpha\left(a_{1}+2 a \frac{L_{1} q\left\|F\left(x_{0}\right)\right\|}{1-q}\right)\right]\left\|F\left(x_{2}\right)\right\| \\
& \leq q\left\|F\left(x_{2}\right)\right\| \leq q^{3}\left\|F\left(x_{0}\right)\right\| .
\end{aligned}
$$

We also have that

$$
\begin{aligned}
\left\|x_{3}-x_{0}\right\| & \leq\left\|x_{3}-x_{2}\right\|+\left\|x_{2}-x_{1}\right\|+\left\|x_{1}-x_{0}\right\| \\
& \leq\left(q^{3}+q^{2}+q\right)\left\|F\left(x_{0}\right)\right\| \\
& =q\left\|F\left(x_{0}\right)\right\| \frac{1-q^{3}}{1-q}<r .
\end{aligned}
$$

That is, $x_{3} \in U\left(x_{0}, r\right)$. Moreover, using (2.6) for $n=2$ as above we have that

$$
\begin{aligned}
\left\|F\left(x_{3}\right)\right\| \leq & \frac{L}{2}\left\|x_{3}-x_{2}\right\|^{2} \\
& +\left(L_{0}\left\|x_{2}-x_{0}\right\|+a_{0}\right)\left\|x_{3}-x_{2}\right\|+\alpha\left(a_{0}+L_{1}\left(\left\|x_{n-1}-x_{0}\right\|+\left\|x_{n}-x_{0}\right\|\right)\right)\left\|F\left(x_{2}\right)\right\| \\
\leq & \frac{L}{2} q^{4}\left\|F\left(x_{0}\right)\right\|\left\|F\left(x_{2}\right)\right\| \\
& +\left(L_{0} \frac{q\left\|F\left(x_{0}\right)\right\|}{1-q}+a_{0}\right) q\left\|F\left(x_{2}\right)\right\|+\alpha\left(a_{0}+2 L_{1} \frac{q\left\|F\left(x_{0}\right)\right\|}{1-q}\right)\left\|F\left(x_{2}\right)\right\| \\
\leq & {\left[\frac{L}{2} q^{4}\left\|F\left(x_{0}\right)\right\|+\left(L_{0} \frac{q\left\|F\left(x_{0}\right)\right\|}{1-q}+a_{0}\right) q+\alpha\left(a_{0}+2 L_{1} \frac{q\left\|F\left(x_{0}\right)\right\|}{1-q}\right)\right]\left\|F\left(x_{2}\right)\right\| } \\
\leq & q\left\|F\left(x_{2}\right)\right\| \leq q^{3}\left\|F\left(x_{0}\right)\right\| .
\end{aligned}
$$

The rest follows in analogous way using induction (simply replace $x_{1}, x_{2}, x_{3}$ by $x_{n-1}, x_{n}, x_{n+1}$ in the preceding estimates). By letting $n \rightarrow \infty$ in (2.5) we obtain $F\left(x^{*}\right)=0$.

Condition $\left(C_{1}\right)$ may not be satisfied but weaker condition $\left(C_{2}\right)$ may be satisfied. In this case condition $\left(C_{1}\right)$ can be dropped. Then, using instead of approximation (2.6) the approximation

$$
\begin{aligned}
F\left(x_{n+1}\right)= & \int_{0}^{1}\left[\left(F^{\prime}\left(x_{n}+\theta\left(x_{n+1}-x_{n}\right)\right)-F^{\prime}\left(x_{0}\right)\right)+\left(F^{\prime}\left(x_{0}\right)-F^{\prime}\left(x_{n}\right)\right)\right]\left(x_{n+1}-x_{n}\right) \mathrm{d} \theta \\
& +\left(F^{\prime}\left(x_{n}\right)-F^{\prime}\left(x_{0}\right)+F^{\prime}\left(x_{0}\right)-A\right)\left(x_{n+1}-x_{n}\right)+ \\
& \alpha_{n}\left(\left[x_{n-1}, x_{n} ; F\right]-F^{\prime}\left(x_{0}\right)+F^{\prime}\left(x_{0}\right)-A\right) F\left(x_{n}\right),
\end{aligned}
$$

we arrive in an analogous way to Theorem 2.1 at the following semilocal convergence result for the Damped Secant method $(1.2)$ under the $\left(C^{0}\right)$ conditions. 
Theorem 2.2 Suppose that the $\left(C^{0}\right)$ conditions hold. Then sequence $\left\{x_{n}\right\}$ generated by the Damped secant method (1.2) is well defined, remains in $\overline{U\left(x_{0}, r\right)}$ for each $n=0,1,2, \ldots$, and converges to a solution $x^{*} \in$ $\overline{U\left(x_{0}, r\right)}$ of equation (1.1). Moveover, the following estimates hold for each $n=0,1,2, \ldots$,

$$
\left\|x_{n+1}-x_{n}\right\| \leq q\left\|F\left(x_{n}\right)\right\| \leq q^{n+1}\left\|F\left(x_{0}\right)\right\|,
$$

and

$$
\left\|F\left(x_{n+1}\right)\right\| \leq q\left\|F\left(x_{n}\right)\right\| \leq q^{n+1}\left\|F\left(x_{0}\right)\right\|
$$

where $q$ is defined in $\left(C_{6}\right)$ and $r$ in $\left(C_{7}\right)$.

Concerning the uniqueness of the solution $x^{*}$ in $\overline{U\left(x_{0}, r\right)}$ we have the following result.

Proposition 2.3 Suppose that the $(C)$ or $\left(C^{0}\right)$ conditions hold. Moveover, suppose that there exist $x_{0} \in D$ and $r_{1} \geq r$ such that $F^{\prime}\left(x_{0}\right)^{-1} \in \mathcal{L}(\mathbb{X}, \mathbb{Y})$ and

$$
\left\|F^{\prime}\left(x_{0}\right)^{-1}\right\| L_{0}\left(r_{1}+r\right)<2 .
$$

Then the solution $x^{*}$ is the only solution of equation (1.1) in $\overline{U\left(x_{0}, r_{1}\right)}$, where $r$ is defined in $\left(C_{7}\right)$.

Proof. The existence of the solution $x^{*}$ is guaranteed by conditions $(C)$ or $\left(C^{0}\right)$. To show uniqueness, let $y^{*} \in \overline{U\left(x_{0}, r_{1}\right)}$ with $F\left(y^{*}\right)=0$. Define $\mathcal{Q}=\int_{0}^{1} F^{\prime}\left(x^{*}+\theta\left(y^{*}-x^{*}\right)\right) \mathrm{d} \theta$. Then, using $\left(C_{2}\right)$ and $(2.8)$ we obtain in turn that

$$
\begin{aligned}
\left\|F^{\prime}\left(x_{0}\right)^{-1}\right\|\left\|\mathcal{Q}-F^{\prime}\left(x_{0}\right)\right\| & \leq\left\|F^{\prime}\left(x_{0}\right)^{-1}\right\| L_{0} \int_{0}^{1}\left\|\left(x^{*}-x_{0}\right)+\theta\left(y^{*}-x^{*}\right)\right\| \mathrm{d} \theta \\
& \leq\left\|F^{\prime}\left(x_{0}\right)^{-1}\right\| L_{0} \int_{0}^{1}\left\|(1-\theta)\left(x^{*}-x_{0}\right)+\theta\left(y^{*}-x_{0}\right)\right\| \mathrm{d} \theta \\
& \leq\left\|F^{\prime}\left(x_{0}\right)^{-1}\right\| \frac{L_{0}}{2}\left(r+r_{1}\right)<1 .
\end{aligned}
$$

It follows from $(2.9)$ and the Banach lemma on invertible operator [11] that $\mathcal{Q}^{-1} \in \mathcal{L}(\mathbb{Y}, \mathbb{X})$. Moreover, we have that $0=F\left(y^{*}\right)-F\left(x^{*}\right)=\mathcal{Q}\left(y^{*}-x^{*}\right)$, which implies $x^{*}=y^{*}$.

\section{Local convergence}

In this section, we present the local convergence analysis of Damped Secant method (1.2). We shall use the following conditions:

$\left(H_{0}\right) \quad F: D \subseteq \mathbb{X} \rightarrow \mathbb{Y}$ is Fréchet-differentiable and there exist $A \in \mathcal{L}(\mathbb{X}, \mathbb{Y}), x^{*} \in D, a \geq 0, \beta \geq 0, c_{0} \geq 0$ such that $A^{-1} \in \mathcal{L}(\mathbb{Y}, \mathbb{X}), F\left(x^{*}\right)=0,\left\|A^{-1}\right\| \leq a,\left\|F^{\prime}\left(x^{*}\right)\right\| \leq \beta$ and $\left\|A^{-1}\left(A-F^{\prime}\left(x^{*}\right)\right)\right\| \leq c_{0}$

$\left(H_{1}\right)$ There exist $L>0$ such that for each $x, y \in D$ the Lipschitz condition

$$
\left\|F^{\prime}(x)-F^{\prime}(y)\right\| \leq L\|x-y\|
$$

holds;

$\left(H_{2}\right)$ There exists $l_{0}>0$ such that for each $x \in D$ the center-Lipschitz condition

$$
\left\|F^{\prime}(x)-F^{\prime}\left(x^{*}\right)\right\| \leq l_{0}\left\|x-x^{*}\right\|
$$

holds;

$\left(H_{3}\right)$ There exist a divided difference $[x, y ; F]$, and $L_{1} \geq 0$ such that

$$
\left\|[x, y ; F]-F^{\prime}\left(x^{*}\right)\right\| \leq L_{1}\left(\left\|x-x^{*}\right\|+\left\|y-x^{*}\right\|\right) ;
$$


$\left(H_{4}\right)$ There exists $\alpha \geq 0$ such that

$$
\left|\alpha_{n}\right| \leq \alpha \quad \text { and } \quad c_{0}(1+\alpha \beta)<1 .
$$

$\left(H_{5}\right)$ Denote by $R_{1}$ the positive root of quadratic polynomial

$$
p_{1}(t)=\frac{\alpha l_{0}^{2}}{2} a t^{2}+\left(\frac{a L}{2}+\frac{\alpha l_{0} c_{0}}{2}+l_{0} a(1+\alpha \beta)+2 a L_{1}+a l_{0}\right) t+c_{0}(1+\alpha \beta)-1 .
$$

Moreover, denote by $R_{2}$ the positive root of quadratic polynomial

$$
p_{2}(t)=\frac{a \alpha l_{0}^{2}}{2} t^{2}+\left(\frac{3 a l_{0}}{2}+\frac{c_{0} a l_{0}}{2}+l_{0} a(1+\alpha \beta)+2 a L_{1}+a l_{0}\right) t+c_{0}(1+\alpha \beta)-1 .
$$

$\left(H_{6}\right) \overline{U\left(x^{*}, R\right)} \subseteq D$, where $R$ is $R_{1}$ or $R_{2}$.

Notice that $\left(H_{1}\right)$ implies $\left(H_{2}\right)$,

$$
l_{0} \leq L
$$

holds in general and $\frac{L}{l_{0}}$ can be arbitrarily large $[3,4,7]$. The quadratic polynomials $p_{1}(\mathrm{t})$ and $p_{2}(t)$ have a positive root by the second hypothesis in $\left(H_{4}\right)$ and the positivity of the coefficients of $t$ and $t^{2}$. From now on we shall denote $\left(H_{0}\right)-\left(H_{6}\right)$ and $\left(H_{0}\right)$ and $\left(H_{2}\right)-\left(H_{6}\right)$ as the $(H)$ and $\left(H^{0}\right)$ conditions, respectively. Next, we present the local convergence of Secant-like method (1.2) first under the $(H)$ conditions. In view of $(1.2)$ and $F\left(x^{*}\right)=0$, we can have the following identity

$$
\begin{aligned}
x_{n+1}-x^{*}= & -A^{-1}\left\{\int_{0}^{1}\left[F^{\prime}\left(x^{*}+\theta\left(x_{n}-x^{*}\right)\right)-F^{\prime}\left(x_{n}\right)\right] \mathrm{d} \theta\right. \\
& -\left(\left(A-F^{\prime}\left(x^{*}\right)\right)+\left(F^{\prime}\left(x^{*}\right)-F^{\prime}\left(x_{n}\right)\right)\right)\left[\left(I-\alpha_{n} F^{\prime}\left(x^{*}\right)\right)\right. \\
& \left.-\alpha_{n} \int_{0}^{1}\left[F^{\prime}\left(x^{*}+\theta\left(x_{n}-x^{*}\right)\right)-F^{\prime}\left(x^{*}\right)\right]\right]+ \\
& \left.\left(\left[x_{n-1}, x_{n} ; F\right]-F^{\prime}\left(x^{*}\right)\right)+\left(F^{\prime}\left(x^{*}\right)-F^{\prime}\left(x_{n}\right)\right)\right\}\left(x_{n}-x^{*}\right) .
\end{aligned}
$$

Then, using (3.2), and the $(H)$ conditions, it is standard to arrive at the following result (see, for example $[9,10,13])$ :

Theorem 3.1 Suppose that the $(H)$ conditions hold. Then sequence $\left\{x_{n}\right\}$ generated by the Damped Secant method (1.2) is well defined, remains in $\overline{U\left(x^{*}, R_{1}\right)}$ for each $n=0,1,2, \ldots$, and converges to $x^{*}$ provided that $x_{0} \in \overline{U\left(x^{*}, R_{1}\right)}$. Moveover, the following estimates hold for each $n=0,1,2, \ldots$,

$$
\left\|x_{n+1}-x^{*}\right\| \leq e_{n}\left\|x_{n}-x^{*}\right\|<\left\|x_{n}-x^{*}\right\|<R_{1},
$$

where

$$
\begin{aligned}
e_{n}= & \frac{a L}{2}\left\|x_{n}-x^{*}\right\|+\left(c_{0}+\alpha l_{0}\left\|x_{n}-x^{*}\right\|\right)\left(1+\alpha \beta+\frac{\alpha l_{0}}{2}\left\|x_{n}-x^{*}\right\|\right) \\
& +a\left(L_{1}\left(\left\|x_{n-1}-x^{*}\right\|+\left\|x_{n}-x^{*}\right\|\right)+l_{0}\left\|x_{n}-x^{*}\right\|\right) \\
< & p_{1}\left(R_{1}\right)+1<1 .
\end{aligned}
$$

In cases $\left(H_{1}\right)$ cannot be verified but $\left(H_{2}\right)$ holds, we can present the local convergence of the Damped Secant method (1.2) under the $\left(H^{0}\right)$ conditions using the following modification of the Ostrowski representation (3.2) given by

$$
\begin{aligned}
x_{n+1}-x^{*}= & -A^{-1}\left\{\int_{0}^{1}\left[F^{\prime}\left(x^{*}+\theta\left(x_{n}-x^{*}\right)\right)-F^{\prime}\left(x^{*}\right)\right] \mathrm{d} \theta+\left[F^{\prime}\left(x^{*}\right)-F^{\prime}\left(x_{n}\right)\right]-\left(\left(A-F^{\prime}\left(x^{*}\right)\right)+\right.\right. \\
& \left.\left(F^{\prime}\left(x^{*}\right)-F^{\prime}\left(x_{n}\right)\right)\right)\left[\left(I-\alpha_{n} F^{\prime}\left(x^{*}\right)-\alpha_{n} \int_{0}^{1}\left[F^{\prime}\left(x^{*}+\theta\left(x_{n}-x^{*}\right)\right)-F^{\prime}\left(x^{*}\right)\right]\right]+\right. \\
& \left.\left(\left[x_{n-1}, x_{n} ; F\right]-F^{\prime}\left(x^{*}\right)\right)+\left(F^{\prime}\left(x^{*}\right)-F^{\prime}\left(x_{n}\right)\right)\right\}\left(x_{n}-x^{*}\right) .
\end{aligned}
$$


Theorem 3.2 Suppose that $\left(H^{0}\right)$ conditions hold. Then sequence $\left\{x_{n}\right\}$ generated by the Damped Secant method $(1.2)$ is well defined, remains in $\overline{U\left(x^{*}, R_{2}\right)}$ for each $n=0,1,2, \ldots$, and converges to $x^{*}$ provided that $x_{0} \in$ $\overline{U\left(x^{*}, R_{2}\right)}$. Moveover, the following estimates hold for each $n=0,1,2, \ldots$,

$$
\left\|x_{n+1}-x^{*}\right\| \leq e_{n}^{0}\left\|x_{n}-x^{*}\right\|<\left\|x_{n}-x^{*}\right\|<R_{2}
$$

where

$$
\begin{aligned}
e_{n}^{0}= & \frac{3 l_{0} a}{2}\left\|x_{n}-x^{*}\right\|+\left(c_{0}+\alpha l_{0}\left\|x_{n}-x^{*}\right\|\right)\left(1+\alpha \beta+\frac{\alpha l_{0}}{2}\left\|x_{n}-x^{*}\right\|\right) \\
& +a\left(L_{1}\left(\left\|x_{n-1}-x^{*}\right\|+\left\|x_{n}-x^{*}\right\|\right)+l_{0}\left\|x_{n}-x^{*}\right\|\right) \\
< & p_{2}\left(R_{2}\right)+1<1 .
\end{aligned}
$$

\section{Numerical Examples}

In this section we are going to present some examples in order to analyze the different conditions and bounds for the semilocal and local convergence and to confirm the theoretical results.

Example 1 In this example we consider the $\mathbb{X}=\mathbb{Y}=\mathbb{R}$ and the function $F(x)=x^{3}-0.49$ defined in $[0,1]$. Let us consider $x_{0}=0.85, A=F^{\prime}\left(x_{0}\right)$ and $\alpha_{n}=0.001$, for all $n \geq 0$. So, $a=0.4614, a_{0}=a_{1}=0$ and $\alpha=0.001$. For this example, the constant of Lipschitz conditions are $L=6, L_{0}=5.55$ and $L_{1}=2.775$. Then, in order to satisfy conditions (2.1) and (2.2) of $\left(C_{5}\right)$, we need to choose $q=0.5369$. Therefore, $r=0.1439$ and, from Theorem 2.1, the ball of convergence is $\overline{U\left(x_{0}, r\right)}$.

Example 2 Let $\mathbb{X}=\mathbb{Y}=\mathbb{R}^{3}$ and the function $F: D \subseteq \mathbb{R}^{3} \rightarrow \mathbb{R}^{3}$ defined as

$$
F(x, y, z)=\left(x, y^{2}+y, e^{z}-1\right) .
$$

Then, the Fréchet derivative of $F$ is given by

$$
F^{\prime}(x, y, z)=\left(\begin{array}{ccc}
1 & 0 & 0 \\
0 & 2 y+1 & 0 \\
0 & 0 & e^{z}
\end{array}\right)
$$

Notice that $x^{*}=(0,0,0)^{T}$ is a root of the system $F(x, y, z)=0$. Let us consider the domain $D=\overline{U\left(x^{*}, 0.5\right)}$, $A=1.43 I$, where $I$ denotes the identity matrix, $x_{0}=(0.1,0.1,0.1)^{T}, \alpha_{n}=0$ for all $n \geq 0$. So, a=0.6993, $a_{0}=0.57$ and $a_{1}=a_{0} a=0.3007$, and $\alpha=0$.

Example 3 Now, we consider the $\mathbb{X}=\mathbb{Y}=\mathbb{R}$ and the function $F(x)=x^{3}-2$ in $D=[0.75,1.75]$, whose real root is $x^{*}=1.25992 \ldots$. We take $A=4.8$ (so, $a=0.2083$ ), $\beta=4.7622, c_{0}=0.00787, l_{0}=7.6798, L_{1}=4.7622$ and $\alpha_{n}=1 / 1000, n=0,1,2, \ldots$ So, $\alpha=0.001$ and applying conditions $\left(H^{0}\right)$, the roots of polynomial $p_{2}(t)$ are $t_{1}=0.115258$ and $t_{2}=-1006.79$. From Theorem 3.2, the ball of convergence is $\overline{U\left(x^{*}, t_{1}\right)}$. By choosing

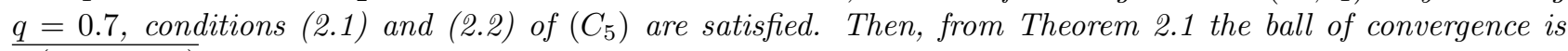
$\overline{U\left(x_{0}, 0.2455\right)}$

Example 4 Let $\mathbb{X}=\mathbb{Y}=\mathbb{R}^{3}$ and the function $F: D \subseteq \mathbb{R}^{3} \rightarrow \mathbb{R}^{3}$ defined as

$$
F(x, y, z)=\left(e^{x}-1, \frac{e-1}{2} y^{2}+y, z\right) .
$$

Then, the Fréchet derivative of $F$ is given by

$$
F^{\prime}(x, y, z)=\left(\begin{array}{ccc}
e^{x} & 0 & 0 \\
0 & (e-1) y+1 & 0 \\
0 & 0 & 1
\end{array}\right)
$$

As $x^{*}=(0,0,0)^{T}$, we have $F\left(x^{*}\right)=(0,0,0)^{T}$ and $F^{\prime}\left(x^{*}\right)=\operatorname{diag}\{1,1,1\}$. If we take $D=\overline{U\left(x^{*}, 1\right)}$, $A=$ $\operatorname{diag}\{1.25,1.25,1.25\}$ and $\alpha_{n}=5 / 100, n=0,1, \ldots$, then the bounds of conditions $\left(H_{0}\right)-\left(H_{4}\right)$ are the following: 
$a=\left\|A^{-1}\right\|=1 / 1.25=0.8, \beta=\left\|F^{\prime}\left(x^{*}\right)\right\|=1, c_{0}=\left\|A^{-1}\left(A-F^{\prime}\left(x^{*}\right)\right)\right\|=|1-1 / 1.25|=0.2$, the constant of the Lipschitz condition is $L=e$, and the constant of the center-Lipschitz condition is $l_{0}=e-1$.

With these bounds the condition $c_{0}(1+\alpha \beta)<1$ is satisfied, and polynomial $p_{1}(t)$ has the roots $t_{1}=-93.5199$, $t_{2}=0.143055$ and $p_{2}(t)$ has the roots $t_{1}=-112.184, t_{2}=0.119256$. Therefore, if we assume $(H)$ conditions, then the ball of convergence is $\overline{U\left(x^{*}, 0.143055\right)}$, whereas that if we assume $\left(H^{0}\right)$ conditions, then the ball of convergence is $\overline{U\left(x^{*}, 0.119256\right)}$.

\section{References}

[1] S. Amat, S. Busquier, and V. Candela, Third-order iterative methods without using any Fréchet derivative, Journal of Computational and Applied Mathematics 158 (2003), pp. 11-18.

[2] S. Amat, S. Busquier, and J.M. Gutiérrez, Geometric constructions of iterative functions to solve nonlinear equations, J. Comput. Appl. Math., 157 (2003), pp. 197-205.

[3] I.K. Argyros, Convergence and applications of Newton-type iterations, Springer-Verlag Publ., New York, 2008.

[4] I. K. Argyros and J. Chen, Improved results on estimating and extending the radius of an attraction ball, Appl. Math. Lett. 23 (2010), pp. 404-408.

[5] I. K. Argyros and J. Chen, On local convergence of a Newton-type method in Banach space, Int. J. Comput. Math. 86 (2009), pp. 1366-1374.

[6] I.K. Argyros and S. Hilout, Improved local convergence of Newton's method under weak majorant condition, J. Comput. Appl. Math., 236 (2012), pp. 1892-1902.

[7] I.K. Argyros and S. Hilout, Computational methods in Nonlinear Analysis, World Scientific Publ. Comp. New Jersey, 2013.

[8] Chandrasekhar, S., Radiative transfer, Dover Publ., New York, 1960.

[9] J. Chen and Q. Sun, The convergence ball of Newton-like methods in Banach space and applications, Taiwanese J. Math. 11 (2007), pp. 383-397.

[10] J. Chen and W. Li, Convergence behaviour of inexact Newton methods under weak Lipschitz condition, J. Comput. Appl. Math. 191 (2006), pp. 143-164.

[11] L.V. Kantorovich and G.P. Akilov, Functional Analysis, Pergamon Press, Oxford, 1982.

[12] D. Herceg, N. Krejić, and Z. Lužanin, Quasi-Newton's method with correction, Novi Sad J. Math., 26 (1996), pp. 115-127.

[13] N. Krejić and Z. Lužanin, Newton-like method with modification of the right-hand-side vector, Math. Comp. 71 (2002), pp. 237-250

[14] M. Petković, B. Neta, L. Petković and J. Džunić, Multipoint methods for solving nonlinear equations, Academic Press, 2013.

[15] F.A. Potra, Sharp error bounds for a class of Newton-like methods, Libertas Mathematica, 5 (1985), pp. $71-84$.

[16] P.D. Proinov, General local convergence theory for a class of iterative processes and its applications to Newton's method, J. Complexity, 25 (2009), pp. 38-62. 